Published in final edited form as:

Sci Transl Med. 2019 August 07; 11(504): . doi:10.1126/scitranslmed.aax7392.

\title{
Personalized circulating tumor DNA analysis to detect residual disease after neoadjuvant therapy in breast cancer
}

\author{
Bradon R. McDonald ${ }^{1}$, Tania Contente-Cuomo ${ }^{1}$, Stephen-John Sammut ${ }^{2}$, Ahuva \\ Odenheimer-Bergman ${ }^{1}$, Brenda Ernst ${ }^{3}$, Nieves Perdigones ${ }^{1}$, Suet-Feung Chin $^{2}$, Maria \\ Farooq $^{1}$, Rosa Mejia ${ }^{4}$, Patricia A. Cronin ${ }^{3}$, Karen S. Anderson ${ }^{3,4}$, Heidi E. Kosiorek ${ }^{3}$, Donald \\ W. Northfelt ${ }^{3}$, Ann E. McCullough ${ }^{3}$, Bhavika K. Patel ${ }^{3}$, Jeffrey N. Weitzel ${ }^{5}$, Thomas P. \\ Slavin $^{5}$, Carlos Caldas ${ }^{2}$, Barbara A. Pockaj ${ }^{3,{ }^{*}}$, Muhammed Murtaza ${ }^{1,3,{ }^{*}}$ \\ ${ }^{1}$ Center for Noninvasive Diagnostics, Translational Genomics Research Institute, Phoenix, AZ \\ 85004, USA. \\ 2Department of Oncology and Cancer Research UK Cambridge Institute and Cancer Centre, Li \\ Ka Shing Centre, University of Cambridge, Cambridge CB2 ORE, United Kingdom. \\ ${ }^{3}$ Mayo Clinic, Scottsdale, AZ 85259, USA. \\ ${ }^{4}$ Biodesign Institute, Arizona State University, Tempe, AZ 85281, USA. \\ ${ }^{5}$ City of Hope, Duarte, CA 91010, USA.
}

\begin{abstract}
Longitudinal analysis of circulating tumor DNA (ctDNA) has shown promise for monitoring treatment response. However, most current methods lack adequate sensitivity for residual disease detection during or after completion of treatment in non-metastatic cancer patients. To address this gap and to improve sensitivity for minute quantities of residual tumor DNA in plasma, we have developed targeted digital sequencing (TARDIS) for multiplexed analysis of patient-specific cancer mutations. In reference samples, by simultaneously analyzing 8-16 known mutations, TARDIS achieved $91 \%$ and 53\% sensitivity at mutant allele fractions (AFs) of 3 in $10^{4}$ and 3 in $10^{5}$, respectively, with $96 \%$ specificity, using input DNA equivalent to a single tube of blood. We successfully analyzed up to 115 mutations per patient in 80 plasma samples from 33 women with
\end{abstract}

*Correspondence to: Dr. Muhammed Murtaza (mmurtaza@tgen.org) or Dr. Barbara Pockaj (pockaj.barbara@mayo.edu). Author contributions: BAP and MM conceptualized and designed the study. BRM, TCC, NP and MM developed methods. SJS, BE, PAC, KSA, HEK, DWN, AEM, BKP, JNW, TPS, CC and BAP designed and conducted the prospective clinical studies. TCC, SJS, SFC, AOB, MF and RM generated data. BRM and MM analyzed sequencing data. BRM, SJS, TPS, CC, BAP and MM interpreted data. BRM and MM wrote the paper the assistance from SJS, TPS, AOB, CC, BAP and other authors. All authors approved the final manuscript.

Competing interests: MM, TCC, BRM, AOB and NP are inventors or co-inventors on patent applications covering technologies described here including patent application numbers WO2017205540A1 and US201662343802P, both titled "Molecular tagging methods and sequencing libraries" and 62/866,543, titled "Detection and treatment of residual disease using circulating tumor DNA analysis". MM serves as an expert witness in intellectual property litigation related to cell-free DNA analysis methods. CC is a member of AstraZeneca's External Science Panel and is a recipient of research grants (administered by the University of Cambridge) from AstraZeneca, Genentech, Roche and Servier. All other authors declare that they have no competing interests.

Data and materials availability: Analytical performance data from reference samples has been deposited with unrestricted access in Sequence Read Archive (SRA) with accession number PRJNA551456 and will become available upon publication. Targeted sequencing data and tumor/germline exome sequencing data from patient samples will be made available upon reasonable request. All other data associated with this study are present in the paper or Supplementary Materials. 
stage I-III breast cancer. Before treatment, TARDIS detected ctDNA in all patients with $0.11 \%$ median AF. After completion of neoadjuvant therapy, ctDNA concentrations were lower in patients who achieved pathological complete response (pathCR) compared to patients with residual disease (median AFs $0.003 \%$ and $0.017 \%$, respectively, $\mathrm{p}=0.0057, \mathrm{AUC}=0.83$ ). In addition, patients with pathCR showed a larger decrease in ctDNA concentrations during neoadjuvant therapy. These results demonstrate high accuracy for assessment of molecular response and residual disease during neoadjuvant therapy using ctDNA analysis. TARDIS has achieved up to 100-fold improvement beyond current limit of ctDNA detection using clinically relevant blood volumes, demonstrating that personalized ctDNA tracking could enable individualized clinical management of cancer patients treated with curative intent.

\section{One Sentence Summary:}

A robust personalized ctDNA test, TARDIS, achieves high accuracy for residual disease after completion of neoadjuvant therapy.

\section{Introduction}

To maximize the rate of cure, cancer patients with non-metastatic disease are often treated with multiple modalities including pre-operative systemic therapy, surgery and radiation therapy, and post-operative therapy. However, for some patients, this results in overtreatment and adverse effects when they could have been cured with less intensive treatment and the benefit of each consecutive modality of therapy is not certain(1). A treatment monitoring biomarker that can accurately distinguish residual disease from disease eradication could enable individualized management of localized cancers, but this has remained elusive because current diagnostics have inadequate sensitivity. In breast cancer, 30\% patients treated with neoadjuvant therapy achieve pathological complete response (pathCR) with no histological evidence of invasive tumor in the resected breast tissue and lymph nodes(2). pathCR during neoadjuvant therapy is associated with excellent long-term clinical outcomes. Ten-year relapse-free survival rates are $95 \%, 86 \%$, and $83 \%$ in patients with human epidermal growth factor receptor 2-positive (HER2+), triple negative, and estrogen receptorpositive, human epidermal growth factor receptor 2-negative (ER+HER2-) breast cancer who achieve pathCR, respectively(3). In these patients, the added value of surgery, beyond removing pre-neoplastic lesions and confirming pathCR, might be questioned. An alternative diagnostic test to accurately detect residual disease could guide choice and planning of local treatment options such as the extent of surgical resection or the use of radiation therapy (4, $5)$.

Recent advances in circulating tumor DNA (ctDNA) analysis have shown promise in monitoring non-metastatic cancer patients, but these have primarily focused on recurrence monitoring and have limited accuracy for residual disease detection during treatment(6-9). In particular, detection of ctDNA after completion of neoadjuvant therapy has been challenging in patients with breast and rectal cancer, even when residual disease is observed at the time of surgery. Recent studies have found that ctDNA becomes undetectable in more than $90 \%$ of patients during neoadjuvant therapy(10). As a result, no association has been observed between ctDNA detection and pathCR $(11,12)$. Detection of low amounts of 
ctDNA in non-metastatic cancer patients is impeded by limited blood volumes accessible in a clinical environment and low concentrations of total cell-free DNA (cfDNA). Unlike in patients with metastatic cancer where cfDNA concentrations are much higher, a $10 \mathrm{ml}$ blood tube (4 ml plasma) from early stage cancer patients typically yields only $20 \mathrm{ng}$ cfDNA ( 6000 haploid genome copies). In addition, ctDNA concentrations in early and locally advanced cancer patients are lower compared to metastatic cancer patients. For example, median ctDNA concentration before treatment in triple negative breast cancer (TNBC) was $12.5 \%$ in metastatic cancer patients and $0.68 \%$ in non-metastatic cancer patients (almost 20 fold lower) $(12,13)$. During and after completion of treatment, ctDNA signal from residual disease is expected to be even lower. As a result, sensitivity and analytical precision of ctDNA tests for residual disease are often limited due to stochastic sampling variation (Fig. $1 \mathrm{~A})$.

Sampling variation can be overcome by increasing the volume of blood obtained at each time point to increase the amount of plasma DNA analyzed, by improving the rate of conversion of DNA into sequencing-ready molecules, and by simultaneously analyzing multiple patient-specific somatic founder mutations. Founder mutations are present in all cancer cells and therefore, each is equally informative of tumor-derived DNA in blood(14). To leverage these principles and enable residual disease detection, we have developed a personalized approach for tumor-guided ctDNA detection and quantification called targeted digital sequencing (TARDIS). Here, we describe development and analytical performance of TARDIS using dozens of replicates of reference material with tumor fractions as low as 3 in $10^{5}$, and we demonstrate clinical performance of ctDNA detection and quantification in patients with early and locally advanced breast cancer, before and after completion of neoadjuvant systemic therapy.

\section{Results}

\section{Tumor-guided ctDNA analysis using TARDIS}

We developed TARDIS to improve analytical sensitivity and quantitative precision for ctDNA analysis by maximizing interrogation of tumor-derived DNA fragments in limited amounts of plasma DNA. To achieve this, we leveraged simultaneous deep sequencing of patient-specific somatic mutations while minimizing template DNA losses during library preparation and suppressing background errors. For each patient, we identified putative founder somatic mutations using exome sequencing of tumor biopsies and analyzed dozens to hundreds of mutations simultaneously in serial plasma DNA samples obtained during treatment (fig. S1). To maximize capture and analysis of input DNA while preserving specificity, we perform targeted linear pre-amplification, followed by single-stranded DNA ligation with unique molecular identifiers (UMIs), targeted exponential PCR, and sequencing (Fig. 1B). The resulting sequencing reads at each targeted locus have a fixed amplification end and a variable ligation end, preserving fragment size information unlike conventional PCR amplicons $(15,16)$. We used fragment sizes and UMIs to group sequencing reads into read families (RFs) and required consensus of all members to distinguish true low abundance mutations from polymerase or sequencing background errors (Fig. 1C). 


\section{Evaluation of assay performance in reference samples}

To evaluate the analytical performance of TARDIS at low ctDNA concentrations, we designed a multiplexed panel targeting 8 mutations in commercially available reference samples for cfDNA analysis (Seraseq ctDNA Mutation Mix v2, Seracare, table S1). We analyzed a total of 93 replicates, $7-16$ each at $1 \%, 0.5 \%, 0.25 \%, 0.125 \%, 0.063 \%, 0.031 \%$ allele fractions (AFs) and 16 wild-type (WT) samples. AFs for individual mutations were verified by droplet digital PCR (ddPCR) by the vendor (except for $0.063 \%$ and $0.031 \%$ that were dilutions of $0.125 \%$ in WT, table S2). Input DNA in each replicate was 5.6-7.9 ng (1682-2394 haploid genomic equivalents). Mean number of mutated molecules expected for each targeted mutation in a sample was $0.90-19.6$ across $0.031 \%-1 \%$ AFs.

To exclude polymerase errors introduced during linear or exponential amplification, we required at least two independent DNA fragments ( $\geq 2 \mathrm{RFs}$ ) and measured AF consistent with $\searrow 0.5$ mutant molecules to support each variant call. In raw sequencing results, we observed a mean error rate per base of $6.4 \times 10^{-4}$ and median error rate per base of $2.2 \times 10^{-4}$, with background errors observed at $77 \%$ of tested positions. By requiring consensus of all members of an RF and a minimum of $2 \mathrm{RFs}$, we found a significantly reduced mean error rate of $1.1 \times 10^{-4}$ and a median of 0 (Wilcoxon rank-sum $\mathrm{p}<1 \times 10^{-99}$, fig. S2). $93 \%$ of tested positions were error-free. In reference samples, we achieved mutation-level sensitivity of $94.6 \%, 90.6 \%, 65.6 \%, 50.8 \%, 25.8 \%$, and $19.6 \%$ at $1 \%, 0.5 \%, 0.25 \%, 0.125 \%, 0.063 \%$, and $0.031 \% \mathrm{AFs}$, respectively, consistent with the decreasing number of mutant molecules at lower AFs (Fig. 2A, data file S1). Using the same criteria, none of the 128 candidate mutations were detected in WT samples (100\% specificity). Analogous to the detection of tumor-derived DNA in plasma, we leveraged multiple mutations to evaluate sample-level sensitivity. To ascertain mutant DNA in a sample, we required $\geq 2 \mathrm{RFs}$ contributed by one or more mutations, each with measured AF consistent with $\searrow 0.5$ mutant molecules in input DNA. In samples where a single mutation was detected, we required supporting RFs with $\geq 2$ fragment sizes. We achieved sample-level sensitivity of $100 \%$ for $0.125 \%-1 \%$ AFs, $87.5 \%$ for $0.063 \%$, and $78.6 \%$ for $0.031 \%$ AF (Fig. 2B). Using the same criteria, we detected mutant DNA in 1 of 16 wild-type samples (93.8\% specificity). These results demonstrate the principle underlying TARDIS: leveraging multiple patient-specific mutations to overcome limits of sampling and to improve limit of detection. We successfully detected mutant DNA in 11 of 14 replicates with $0.031 \%$ AF with $7.8 \mathrm{ng}$ input DNA per reaction, when we expected a total of 7.2 mutant molecules per reaction across 8 mutations $(<1$ mutant molecule per mutation).

To determine quantitative accuracy, we compared known AFs for variants measured by ddPCR in reference samples to mean AFs measured using TARDIS and found a strong correlation (Pearson $\mathrm{r}=0.921, \mathrm{p}<2.2 \times 10^{-16}$, Fig. $2 \mathrm{C}$ ). To evaluate agreement between observed and expected mutant fraction in each sample (equivalent to ctDNA fraction in plasma samples), we calculated sample-level mean AFs (mean of 8 mutations in each replicate) and found an excellent correlation between observed and expected AFs (Pearson $\mathrm{r}=0.937, \mathrm{p}<2.2 \times 10^{-16}$, Fig. 2D). To evaluate quantitative precision, we calculated the coefficient of variation (CV) for observed AFs for each set of replicates (Fig. 2E). Variantlevel CVs were strongly correlated with expected number of mutated molecules (Pearson $\mathrm{r}=$ 
$\left.-0.854, \mathrm{p}=1.2 \times 10^{-14}\right)$, ranging from 0.28 (17.8 average mutant molecules per mutation) to 3.74 (0.08 mutant molecules per mutation). A similar pattern was observed in CVs for sample-level mean AFs (Pearson $\mathrm{r}=-0.936, \mathrm{p}=0.006$ ), although precision improved when multiple mutations were aggregated together. Sample-level CVs ranged from 0.16 for $1 \%$ expected AF (137.9 average mutant molecules per reaction) to 0.87 for $0.031 \%$ expected $\mathrm{AF}$ (5.4 mutant molecules per reaction, Fig $2 \mathrm{~F}$ ).

To evaluate if we can improve the limit of detection further using clinically accessible amounts of plasma DNA, we performed an additional experiment targeting 16 mutations in 56 replicates from reference samples, 8 replicates each at $1 \%$ and $0.03 \% \mathrm{AFs}$ and wild-type, and 32 replicates at $0.003 \%$ AF. DNA input per reaction was 5.0-13.6 ng for 1\%, $0.03 \%$, and wild-type samples, and 20.0-27.2 ng for $0.003 \%$ AF. Using these input amounts, we expected an average of 38.1, 1.6, and 0.28 mutant molecules per mutation and detected $89.1 \%, 14.1 \%$, and $5.9 \%$ in $1 \%, 0.03 \%$, and $0.003 \%$ AFs, respectively (Fig. 3A, data file S2). Only 1 of 128 candidate mutations was erroneously called as false positive in wild-type samples (99.2\% specificity). Aggregating 16 mutations together, we expected 553, 19.8, and 2.9-4.0 total mutant molecules per sample. At the sample level, we detected tumor DNA in $8 / 8(100 \%), 7 / 8(87.5 \%)$, and $17 / 32(53.1 \%)$ replicates in $1 \%, 0.03 \%$, and $0.003 \%$ AFs, respectively (Fig. 3B). Tumor DNA was not confidently detected in any of 8 wild-type samples using the same criteria (see Materials and Methods). To mimic analysis of cfDNA equivalent to two $10 \mathrm{ml}$ blood tubes (a clinically accessible and relevant volume), we tested all combinations of any 2 replicates at $0.003 \%$ and achieved a sample-level sensitivity of $85.9 \%$ (237/276 combinations). Using a similar approach with wild-type samples, we detected tumor DNA in 1/28 combinations (96.4\% specificity). As in the previous 8 mutations experiment, we observed excellent agreement between sample-level AFs measured using TARDIS and digital PCR (Fig. 3C). Overall, these results confirm quantitative accuracy of TARDIS and demonstrate that simultaneously assaying multiple patient-specific mutations improves sensitivity as well as quantitative precision.

Because limited blood volumes can be obtained clinically, a key performance metric for ctDNA assays is conversion efficiency, or the fraction of input DNA molecules that are successfully analyzed. TARDIS uses several cycles of linear pre-amplification before ligation with UMIs and therefore, we expect the number of read families to be several folds higher than input haploid genome copies. To estimate effective molecular conversion for TARDIS, we leveraged multiple replicates from reference samples and inferred effective conversion by comparing observed performance (sensitivity and precision) and expected performance (based on the Poisson distribution), given expected mutation AFs, amounts of input, and sequencing coverage. Measuring 16 candidate mutations in aggregate, we found that precision improved as the number of total mutant molecules increased in the reaction (Fig. 3D). Theoretically, a linear fit to $\log _{2}(C V)$ as a function of $\log _{2}$ (expected number of mutant molecules) should have a slope of -0.5 , with CVs decreasing as the inverse square root of the number of rare mutant molecules $\left(1 / \bigvee_{h}\right)$ increases. In our data, we observed a slope of -0.496 , consistent with this expectation. Using the intercept for the linear fit and input amounts used for each replicate, we inferred an effective molecular conversion rate of $26 \%$. In contrast, the sample-level sensitivity observed across 32 replicates of $0.003 \%$ suggests effective molecular conversion rate of $\sim 39 \%$, based on expected AFs. 


\section{Detection of residual disease in patients with early and locally advanced breast cancer}

To evaluate whether TARDIS enables residual disease detection in early and locally advanced cancer patients, we analyzed blood samples obtained from 33 patients with Stage I-III breast cancer, of whom 22 patients were treated with neoadjuvant therapy. Distributions of key clinical characteristics of the cohort are presented in Fig. 4A. Most patients presented with Stage II disease (24/33 patients) and invasive ductal carcinoma (30/33 patients). The majority of patients were diagnosed with ER+ HER2- cancer (17/33 patients). $7 / 33$ and 9/33 patients had HER2+ cancer and TNBC, respectively. We performed whole exome sequencing of DNA from diagnostic tumor biopsies and matched germline samples, achieving $177 \mathrm{x}$ and $143 \mathrm{x}$ mean coverage, respectively (table S3). Mean tumor cellularity inferred from exome sequencing analysis was $41 \%$ (range 14\%-85\%). A mean of 700 somatic mutations (range 147-4904) were called across tumor samples, and we identified a mean of 65.8 putative founder mutations per patient (range 9-286). Using an aggressive filtering strategy during primer design and after excluding primers amplifying erroneously in validation experiments with control samples, we analyzed 80 serial plasma samples obtained from 33 patients (1-4 samples per patient, data file S3) for 6-115 mutations per patient (mean 30 and median 18 mutations). Across this cohort, validated TARDIS assays covered a mean of 55\% putative founder mutations found in the corresponding tumor samples (range 5\%-90\%, median 67\%). Plasma samples were collected before starting therapy, during neoadjuvant therapy (NAT), and after completion of NAT before surgery. Input plasma DNA amounts were 4.8-34.5 ng per sample (mean 14.1, median 12.7), obtained from 1.2-4.7 ml plasma (mean 3.8, median 4.0). Total plasma DNA concentration was a median of $5.3 \mathrm{ng} / \mathrm{ml}$ plasma (range 0.83-165.1, mean 8.5). The distribution of total plasma DNA concentration was indistinguishable from healthy volunteers and significantly lower than patients with metastatic cancer (Wilcoxon rank-sum $p=0.0789$ and $p=2.2 \times 10^{-16}$, respectively, fig. S3).

Before treatment, we detected ctDNA in 32/32 patients (Fig. 4B) at tumor fractions of $0.002 \%-1.06 \%$ (mean $0.23 \%$, median $0.11 \%$, Fig. 4C), supported by 2 to 53 distinct mutation events (mean 10.2, median 7.0) and 3 to 1638 mutant RFs (mean 217.5, median 54.5, data file S4). To ensure that analysis of multiple mutations did not increase false positives, we performed multiple-testing correction (Bonferroni correction) and required a corrected $\mathrm{p}<0.05$ for each sample. Baseline plasma sequencing failed in one patient (E009). Plasma samples after completion of neoadjuvant therapy were analyzed in 22 patients. ctDNA was detected in 17/22 patients, including 12/13 patients with invasive or in situ residual disease and 5/9 patients with pathCR (no evidence of tumor cells in the resected tissue). In one patient with residual disease (pathological stage T3 N1 after NAT, patient T065), ctDNA was undetectable in the last blood sample after completion of NAT, likely due to a combination of limited plasma DNA available for analysis (8.7 $\mathrm{ng}$ compared to mean $16.8 \mathrm{ng}$ for samples obtained at similar time points) and limited number of targets analyzed (11 compared to mean of 30 across the entire cohort). We calculated the theoretical maximum number of molecules that could be analyzed for each sample (the product of input haploid genome copies and number of mutations targeted). For patient T065, the maximum number of analyzed molecules in the plasma DNA sample after completion of therapy was 26,273 , the lowest among the post-NAT samples from all patients where the mean was 92,484 molecules. We excluded T065 from further analysis of samples after completion of 
NAT. In patients with detectable ctDNA after NAT, tumor fraction was $0.003 \%-0.045 \%$ (mean $0.018 \%$, median $0.016 \%$ ), supported by 1 to 7 distinct mutation events (mean 3.6, median 4.0) and 2 to 82 mutant RFs (mean 18.8, median 13). Median ctDNA concentrations after completion of NAT were 5.7 fold lower in patients who achieved pathCR compared to patients with residual disease (median AFs $0.003 \%$ vs. $0.018 \%$, respectively, Wilcoxon ranksum one-sided $\mathrm{p}=0.0057$, Fig. 4D). We observed a decrease in ctDNA after NAT compared to pre-treatment concentrations in all but 2 patients (median decrease of $84 \%$, Wilcoxon signed-rank paired one-sided $\mathrm{p}=9.5 \times 10^{-6}$, Fig. 4E). In patients who achieved pathCR, median decrease in ctDNA was $96 \%$, whereas in patients with residual disease observed at surgery, median decrease was 77\% (Wilcoxon rank-sum one-sided $\mathrm{p}=0.055$ ). Temporal changes in variant AFs for multiple mutations within each patient agreed with each other, unless affected by sampling variation as ctDNA decreased during treatment (fig. S4). Using ctDNA concentrations after NAT to differentiate the two groups, we achieved an AUC of $0.83(\mathrm{n}=21$ patients, Fig. 5). The AUC improved to 1 and 0.89 when patients were analyzed as TNBC and ER+ breast cancer sub-groups, respectively ( $\mathrm{n}=9$ and 11 patients, fig. S5).

\section{Discussion}

Patients with early and locally advanced cancers are increasingly treated with neoadjuvant systemic therapy to downstage their tumors and to improve outcomes of subsequent localized treatment. Across some cancer subtypes such as breast, rectal, and esophageal cancers, 20\%-30\% patients achieve pathological complete response after neoadjuvant therapy, which means that no evidence of tumor cells is found in surgically resected tissue (2, $17,18)$. Achieving pathCR is associated with good prognosis, but histopathological evaluation of surgically resected tissue remains the only reliable method to establish pathCR. Imaging and clinical assessment of response have been unable to predict pathCR with high accuracy, and no circulating biomarkers have been informative in this setting $(4,5)$. Our results reveal that ctDNA concentrations after completion of neoadjuvant therapy for breast cancer are higher in patients with residual disease at the time of surgery compared to patients with pathCR.

Several earlier studies have evaluated whether ctDNA analysis can be informative of response to neoadjuvant therapy in breast cancer. However, these studies were limited in sensitivity and precision because ctDNA concentrations in non-metastatic cancer patients are extremely low. In our study, ctDNA was detected in $100 \%$ of early and locally advanced breast cancer patients before treatment (95\% CI 89\%-100\%), improving on earlier reports of $50 \%-75 \%$ ctDNA detection at baseline $(7,19)$. High sensitivity for ctDNA detection before treatment is a pre-requisite for any approach used for residual disease testing because tumor burden is generally higher at presentation. Median pre-treatment ctDNA concentration in our study was $0.11 \%$, about 25-100 times lower than ctDNA concentrations reported in metastatic breast cancer patients $(13,20)$. After completion of neoadjuvant therapy, earlier reports suggest ctDNA concentrations fall below the limit of detection in $>90 \%$ of patients after neoadjuvant therapy, regardless of residual disease status(10-12, 21, 22). In our study, median ctDNA concentrations after neoadjuvant therapy were $0.017 \%$ and $0.003 \%$ in patients with residual disease and pathCR, respectively. 
To achieve sensitivity and quantitative precision required for ctDNA analysis in nonmetastatic cancer patients, we have developed a method for tumor-guided ctDNA analysis that leverages multiple mutations together with improvements in sequencing library preparation and informatics analysis. Earlier studies investigating ctDNA quantification for longitudinal treatment monitoring have targeted single recurrent or patient-specific mutations for plasma DNA analysis using digital PCR or digital sequencing $(8,20)$. Although these approaches are informative of large changes in ctDNA concentration during treatment, ctDNA typically becomes undetectable in mid-treatment samples, even in metastatic cancer patients who have clearly measurable disease on imaging(20). To improve sensitivity and overcome limited input, plasma DNA can be sampled at multiple genomic loci simultaneously. One approach is to analyze a panel of recurrent cancer genes with high coverage targeted sequencing and to integrate results from multiple mutations in each patient $(6,23,24)$. However, this approach typically does not yield more than $2-4$ mutations per patient, limiting the maximum sensitivity achieved regardless of depth of sequencing (Fig. 1A). More recently, analysis of multiple patient-specific mutations pre-identified in the tumor tissue has emerged as an alternative approach, including amplicon sequencing of dozens of mutations, hybrid-capture enrichment of sequencing libraries for dozens to thousands of mutations, and whole genome sequencing. At various stages of development, these approaches generally improve on the current limit of detection for ctDNA analysis ( $\sim .1 \%$ mutation allele fraction), but each approach has some limitations. Conventional multiplexed PCR-based approaches have been limited by the high background error rates observed $(9,22)$. An alternative is to incorporate UMIs during the first few cycles of PCR to overcome background errors, but this limits molecular conversion because template DNA molecules not incorporated within the first 2-3 cycles are excluded from further analysis(16, $25,26)$. In addition, it limits multiplexing capacity and requires optimization for patientspecific assays. In contrast, ligation-based sequencing library preparation enables a wider analysis of the genome but has limited molecular conversion and loses up to $90 \%$ of template DNA molecules due to inefficient ligation(27, 28). Personalized hybrid capture enrichment can overcome this limitation by incorporating thousands of mutations, but such a high number of mutations are only found in a few tumor types, and identifying them requires whole genome sequencing of high-cellularity tumor samples and corresponding normal tissue. Together with synthesis of customized hybrid capture biotinylated baits for each patient, this approach is currently very expensive. An even wider analysis can be performed by whole genome sequencing (WGS) of plasma DNA, either by direct counting of mutated DNA molecules across the genome or by integration of genome-wide patient-specific mutational signatures. However, both approaches require WGS of high-cellularity tumor tissue upfront, and WGS of plasma DNA at the required depth of coverage remains prohibitively expensive for a ctDNA test that may be repeated multiple times during clinical follow-up.

In contrast to efforts highlighted above, TARDIS combines the strengths of PCR-based methods (minimizing losses of template DNA molecules) and ligation-based methods (incorporation of UMIs, preservation of fragment sizes, and hundreds-fold multiplexing). This combination achieves a balance between depth and breadth of tumor genome analyzed, investigating dozens to hundreds of patient-specific mutations with deep coverage. TARDIS 
assays require design, synthesis, and empirical validation of patient-specific primer panels. We have streamlined and automated the design process to successfully target $55 \%$ of putative founder mutations per patient on average. We rely on routine primer synthesis with standard purification and need a limited sequencing footprint, making our approach costeffective and enabling frequent and longitudinal analysis of plasma samples. To identify target mutations, TARDIS requires exome sequencing of tumor DNA from diagnostic tumor biopsies. Compared to WGS, exome sequencing is clinically more feasible in the foreseeable future, generates greater depth of coverage and enables confident identification of putative founder mutations even in lower cellularity tumor samples. At our institution, exome sequencing is routinely performed within 2 weeks of receiving a tumor specimen. Using automated informatics pipelines, a TARDIS assay can be designed, synthesized, and empirically validated for each patient within 1-2 weeks thereafter. Hence, the total turnaround time for development of a patient-specific assay is 3-4 weeks after a diagnostic biopsy, well within the time frame required for clinical decision making for cancer patients treated with neoadjuvant therapy.

To aggregate multiple patient-specific mutations and improve detection sensitivity and quantitative precision for ctDNA analysis, we target founder mutations that are shared by all tumor cells and are equally informative. Sub-clonal mutations are more likely to be lost due to population bottlenecks during treatment and become uninformative for residual disease detection $(9,14)$. Using a combination of founder and subclonal mutations may lower the real world sensitivity of the assay, although tumor specificity will remain unaffected. Similarly, an aggregate ctDNA fraction calculated using a mix of founder and sub-clonal mutations may not reflect true tumor burden. Varying contributions of founder and subclonal mutations can complicate both assessment of longitudinal changes in ctDNA within a patient's clinical course and comparison of ctDNA concentrations across a cohort of patients. Definitive identification of founder mutations requires multisite sequencing, but obtaining multiple biopsies remains clinically challenging. In the current study, we have combined two informatics approaches to maximize the fraction of targeted mutations likely to be founder.

We also report extensive evaluation of analytical performance using commercially available reference samples. Sequencing library preparation typically loses the large majority of input DNA material during early steps such as ligation of adapters. This is particularly challenging for ctDNA analysis because limited blood volumes can be accessed clinically and plasma DNA concentrations are low. We tried to overcome this challenge, while keeping any polymerase-induced errors in check, by using linear pre-amplification of input DNA. To measure our efficiency of molecular conversion, we used an approach based on sensitivity and reproducibility across dozens of replicates of known reference samples. We compared observed sensitivity and precision with expected values based on Poisson distribution and inferred conversion efficiency of $39 \%$ and $26 \%$, respectively. This approach measures effective conversion using real world performance metrics, instead of relying on molecular coverage in the targeted region, a metric that is susceptible to molecular and informatics artifacts due to sequencing and polymerase-induced errors within UMIs and tag-switching (polymerase-induced recombination of UMIs). We propose benchmarking of current and future methods for ctDNA analysis using a similar approach, which can also be applied to 
non-UMI based methods such as conventional amplicon sequencing. The discrepancy between the two conversion estimates for our data likely results from higher estimates of CVs due to variable conversion efficiencies between targeted mutations. These could result from differences in efficiency of linear and exponential amplification, fragment sizes, local secondary structures, and depth of sequencing coverage.

Our results demonstrate potential applications of ctDNA analysis for monitoring response in neoadjuvantly treated cancer patients. We have shown that ctDNA concentrations after completion of neoadjuvant therapy are associated with pathological complete response in early and locally advanced breast cancer. Together with imaging and clinical assessment, ctDNA concentrations may guide treatment strategy in individual patients, such as the choice and extent of local treatment (surgical resection or radiation). The threshold for ctDNA concentrations predictive of residual disease will likely vary between clinical subtypes of breast cancer and between cancer types. Larger clinical studies will be needed to validate our findings and to refine clinically relevant diagnostic thresholds. We have also observed a decrease in ctDNA during neoadjuvant therapy which was greater in magnitude when patients achieve pathCR. This highlights the utility of improved quantitative precision achieved using a multi-mutation assay. Future studies could evaluate whether the magnitude of early decrease in ctDNA concentration during neoadjuvant treatment is informative of therapeutic benefit, enabling adaptive treatment designs to rapidly identify systemic treatment options that work for individual patients. Overall, ctDNA analysis using sensitive and accurate approaches such as TARDIS can enable development of clinical strategies for individualized management of patients treated with curative intent.

A limitation of this study is that we were unable to detect ctDNA in one patient with residual disease after completion of neoadjuvant therapy, despite high volume residual disease. This was most likely due to a combination of low plasma DNA concentration and a limited number of mutations assayed for this patient. ctDNA was detected in this patient in 3 other plasma samples collected before and at 6 and 12 weeks on treatment. Potential approaches to overcome this limitation in future clinical studies include targeting a greater number of putative founder mutations and analyzing larger blood volumes. Although in the current study, we analyzed up to $4 \mathrm{ml}$ plasma obtained from $10 \mathrm{ml}$ blood samples, it is conceivable to collect up to $30 \mathrm{ml}$ blood at a single time point. It is also feasible in future studies to collect and analyze plasma samples over multiple days after completion of therapy to increase sensitivity for residual disease.

Overtreatment of early stage cancer patients remains a challenge in cancer medicine, likely to become more relevant as newer blood- and imaging-based early detection approaches gain credence(29). Most efforts to optimize treatments have focused on tissue-based predictive biomarkers to assess risk of tumor recurrence(30). Our results suggest that blood-based residual disease testing during treatment can further help individualize the choice and extent of each treatment modality. Establishing clinical validity and utility for ctDNA monitoring and residual disease detection will require larger and prospective studies with long-term clinical follow-up. Once validated, using residual disease detection to individualize cancer management could substantially reduce treatment-related morbidity while preserving clinical outcomes. 


\section{Materials and Methods}

\section{Study Design}

The aim of this study was to develop molecular methods to improve limit of detection and quantitative precision for tumor-guided ctDNA analysis. TARDIS was developed and optimized using commercially available reference samples for cell-free DNA analysis. The analytical performance of TARDIS was demonstrated in replicate reactions with known mutation AFs. This was followed by a retrospective proof-of-principle clinical study with prospectively enrolled patients with Stage I-III breast cancer. Prior power analysis, randomization or blinding were not performed for the clinical study.

\section{Patients and samples}

This study includes patients prospectively enrolled at Mayo Clinic, Phoenix, AZ, USA under an approved IRB protocol number 14-006021 (Mayo cohort), at Addenbrooke's Hospital, Cambridge, UK under an approved Research Ethics Committee protocol number 12/EE/ 0484 (Cambridge cohort), and at City of Hope, Duarte, CA, USA under an approved IRB protocol number 96144 (COH cohort). Informed consent was obtained from all patients. Tumor samples obtained at the time of diagnosis were exome sequenced. Blood samples were collected before starting treatment and in a subset of patients, after completion of neoadjuvant therapy before surgical resection. In the Cambridge cohort, additional blood samples were collected at 6 weeks and 12 weeks during neoadjuvant treatment.

\section{DNA extraction from tumor and germline samples}

For the Mayo cohort, tumor DNA was extracted from four $10 \mu \mathrm{m}$ sections obtained from archived formalin-fixed paraffin-embedded tissue using the MagMAX FFPE DNA/RNA Ultra Kit (ThermoFisher Scientific), after macro-dissection to enrich for tumor cells guided by an H\&E stained tumor section. For the Cambridge cohort, tumor DNA was extracted from ten $30 \mu \mathrm{m}$ sections obtained from fresh frozen tumor tissue using the DNeasy Blood and Tissue Kit (Qiagen). Germline DNA was extracted from peripheral blood cells using the DNeasy Blood and Tissue Kit (Qiagen). For the COH cohort, tumor DNA was extracted from five $10 \mu \mathrm{m}$ sections obtained from archived formalin-fixed paraffin-embedded tissue using GeneRead DNA FFPE kit (Qiagen). Germline DNA was extracted from peripheral blood cells using the FlexiGene DNA Kit (Qiagen).

\section{Plasma processing, DNA extraction, and quality assessment}

For the Mayo and Cambridge cohorts, blood was collected in $10 \mathrm{ml}$ K2 EDTA tubes and centrifuged at $820 \mathrm{~g}$ for 10 minutes within 3 hours of venipuncture to separate plasma. $1 \mathrm{ml}$ aliquots of plasma were centrifuged a second time at $16000 \mathrm{~g}$ for 10 minutes to pellet any remaining leukocytes, and the supernatant plasma was stored at $-80^{\circ} \mathrm{C}$. For the $\mathrm{COH}$ cohort, blood was collected in Streck cell-free BCT tubes (Streck) and centrifuged twice to separate plasma. The first spin was at $1600 g$ for 15 minutes at $25^{\circ} \mathrm{C}$. The plasma was then aliquoted and centrifuged again for 10 minutes at $2500 \mathrm{~g}$ at $25^{\circ} \mathrm{C}$. cfDNA was extracted using either the QIAsymphony DSP Circulating DNA Kit (Qiagen) or MagMAX Cell-Free 
DNA Isolation kit (ThermoFisher Scientific). All cfDNA samples were evaluated for yield and quality using droplet digital PCR, as described previously(31).

\section{Tumor/Germline Exome Sequencing}

For the Mayo and $\mathrm{COH}$ cohorts, tumor/germline exome sequencing libraries were prepared using the KAPA Hyper Prep Kit following manufacturer's instructions. Exome enrichment through hybridization was performed using a customized version of Agilent SureSelect V6 exome. For the Cambridge cohort, tumor and germline exome libraries were generated using the Illumina Nextera Rapid Capture Exome Library Preparation kit. We pooled exome libraries and sequenced on Illumina HiSeq or NovaSeq.

\section{Variant calling in tumor exomes and identification of target mutations}

Reads were aligned to human genome version hg19 using bwamem(32), followed by base recalibration using GATK(33), duplicate identification using Picard tools MarkDuplicates, and indel realignment using GATK. Germline mutations were inferred using GATK HaplotypeCaller and Freebayes(34). Somatic tumor mutations were called using MuTect(35), Seurat(36), and Strelka(37). Somatic mutations with an allele frequency $<5 \%$ were removed.

\section{Identification of Putative Founder Mutations}

Potential target mutations found on autosomes were assessed for copy number, purity, and variant allele frequency (VAF). We used Sequenza to infer both the proportion of tumor cells in the sequenced tumor DNA sample and copy number alterations in the tumor(38). For each mutation, the mean variant allele frequency from the variant callers, sample purity, and local copy number were used to infer its cancer cell fraction (CCF) via two different methods: an implementation of the algorithm from McGranahan et al.(39) and PyClone(40). For each sample, the VAF, minor and major copy number, and purity were used as input for PyClone analysis with 25,000 iterations, including 10,000 iterations of burn in.

Founder mutations were identified using a set of criteria for mutation confidence and maximum CCF. To quality as a target for ctDNA analysis, a mutation must have been identified by at least 2 somatic mutation callers, have a mean germline coverage of $>20$ reads and tumor coverage of $>50$ reads passing each mutation caller's filters, and a germline $\mathrm{VAF}<0.01 \%$. In addition, the upper range of the CCF distribution calculated using the McGranahan et al. approach must be equal to 1.0, and the mutation must be found in the highest CCF PyClone mutation cluster.

\section{Primer Design for TARDIS}

Mutations that passed the filtering steps above were used as targets for TARDIS primer design. The primer design process was focused on maximizing TARDIS performance and minimizing spurious amplification, particularly in the linear pre-amplification stage. We first generated primers on the forward or reverse strands up to $350 \mathrm{bp}$ from the target mutation position for both linear and exponential amplification reactions (Primers 1 and 2 for each targeted locus)(41). Primer 1 melting temperature (Tm) range was set to $68-74{ }^{\circ} \mathrm{C}$, and Primer $2 \mathrm{Tm}$ range was $56-60{ }^{\circ} \mathrm{C}$, with Primer 1 upstream and a maximum of 3 bp overlap 
allowed between Primers 1 and 2. During primer selection, we minimized the distance between the 3' end of Primer 2 and the target mutation position, to ensure that short mutant molecules are captured efficiently. To avoid erroneous variants caused by primer synthesis overhangs, we also required a minimum 3 bp distance to the target mutation. To avoid unintended amplification in multiplexed PCR reactions, we used a combination of in silico PCR, sequence comparison to the genome using LAST(42), and 3' primer kmer matching to identify problematic primers for multiplexing. Primer 1s with more than 2 LAST matches outside the target region were excluded, along with Primer 2s with any LAST off-target matches. All combinations of potential Primer 1s were analyzed using in silico PCR. Next, we built a graph in which nodes represented primers and edges linked pairs of primers with predicted PCR products. The nodes were sorted by number of edges, and we iteratively removed the node with the most edges if it was not the last Primer 1 for a given target. This process continued until there were no remaining edges or until all targets only had a single Primer 1 remaining. If there were multiple remaining Primer 1s for a given target, the one with the fewest kmer matches to other target regions was selected. This process was repeated for Primer 2s, except the best primer after graph analysis was selected based on minimizing distance to the target mutation rather than kmer matches. A test run of TARDIS using each primer panel was conducted with 8 replicates of sheared genomic DNA before analyzing plasma samples to identify any remaining problematic primers. A target was removed from the panel before analysis of plasma samples if median proportion $>0.5$ or maximum proportion $>0.75$ of the reads for that target were masked or if the target captured a median proportion $>0.5$ or maximum proportion $>0.75$ of all reads in any control run. For finalized TARDIS assays, mean distance between 3' end of Primers 1 and 2 and the target locus was 56.0 and $31.0 \mathrm{bp}$, respectively (median: 55.0 and $27.0 \mathrm{bp}$ and standard deviation: 17.5 and $13.2 \mathrm{bp}$, respectively).

\section{Preparation of TARDIS sequencing libraries}

TARDIS sequencing libraries were prepared using target-specific linear pre-amplification, ligation, 1-2 rounds of target-specific exponential amplification, and barcoding PCR.

TARDIS reactions were set up using up to $20 \mathrm{ng}$ of template plasma DNA in $10 \mu \mathrm{l}$ volume for linear pre-amplification. For some plasma samples, input DNA was split into two TARDIS reactions and the results were combined informatically. For each TARDIS run, patient-specific primers were pooled equimolarly. For pre-amplification, each Primer 1 pool was used at a final concentration of $0.5-1.0 \mu \mathrm{M}$ (regardless of the number of primers in the panel). Linear pre-amplification was performed using Kapa HiFi HotStart ReadyMix (Kapa Biosystems) at the following thermocycling conditions: $95^{\circ} \mathrm{C}$ for 5 minutes followed by 50 cycles at $98{ }^{\circ} \mathrm{C}$ for 20 seconds, $70{ }^{\circ} \mathrm{C}$ for 15 seconds, $72{ }^{\circ} \mathrm{C}$ for 15 seconds, and $72{ }^{\circ} \mathrm{C}$ for 1 minute. This reaction was followed by a magnetic bead cleanup (SPRIselect, Beckman Coulter) at 1.8x ratio after addition of $10 \%$ ethanol. Pre-amplified DNA was eluted in $10 \mu \mathrm{l}$ water. After dephosphorylation using FastAP (ThermoFisher Scientific), $0.8 \mu \mathrm{l}$ of $100 \mu \mathrm{M}$ ligation adapter was added to each sample. The sequence of the hairpin oligonucleotide used for single-stranded DNA ligation is provided in table S4 and was adapted from Kwok et al. (43). Samples were denatured at $95^{\circ} \mathrm{C}$ for 5 minutes and immediately transferred to an ice bath for at least 2 minutes. We set up ligation reactions using $2.5 \mu \mathrm{l}$ 10x T4 DNA Ligase buffer (New England Biolabs), $2.5 \mu \mathrm{l}$ of $5 \mathrm{M}$ betaine, 2,000 U of T4 DNA ligase (New 
England Biolabs), and $5.8 \mu \mathrm{l}$ of 40\%-60\% PEG8000. Ligation was performed at $16{ }^{\circ} \mathrm{C}$ for 16-24 hours. A magnetic bead cleanup (SPRIselect) was performed at 1x buffer ratio after initially diluting the sample by adding $20-40 \mu \mathrm{l}$ water (to reduce effective PEG concentration during cleanup). An additional dephosphorylation was performed using FastAP.

Exponential PCR was performed in two rounds. In both rounds, a universal reverse primer was used, complementary to the ligated adapter and upstream of the UMI (see table S4 for primer sequences). On the target-specific end, Primer 1 pools were used for the first round and Primer 2 pools were used for the second round. When the total number of targeted mutations exceed 30, $2 \mu \mathrm{l}$ of amplified DNA from round 1 was split across multiple round 2 reactions of $\sim 30$ targets each. In a subset of samples, only the second round of exponential amplification was performed using total ligated DNA. Primers were pooled equimolarly and used at a final pool concentration of $0.5 \mu \mathrm{M}$. Round 1 amplification was performed using Kapa HiFi HotStart ReadyMix with the following thermocycling conditions: $95^{\circ} \mathrm{C}$ for 5 minute followed by 5 cycles at $98^{\circ} \mathrm{C}$ for 20 seconds, $65^{\circ} \mathrm{C}$ for 2 minutes, and 15 cycles at $98{ }^{\circ} \mathrm{C}$ for 20 seconds, $65^{\circ} \mathrm{C}$ for 15 seconds and $72{ }^{\circ} \mathrm{C}$ for 15 seconds, followed by a 1 minute incubation at $72{ }^{\circ} \mathrm{C}$. Round 2 amplification was performed using NEBNext Q5 Hot Start HiFi PCR Mastermix (New England Biolabs) with the following thermocycling conditions: $98{ }^{\circ} \mathrm{C}$ for 1 minute followed by 5 cycles at $98{ }^{\circ} \mathrm{C}$ for 10 seconds, $61.5^{\circ} \mathrm{C}$ for 4 minutes, and 15 cycles at $98{ }^{\circ} \mathrm{C}$ for 10 seconds, $61.5^{\circ} \mathrm{C}$ for 30 seconds, and $72{ }^{\circ} \mathrm{C}$ for 20 seconds, followed by a 2 minute incubation at $72{ }^{\circ} \mathrm{C}$. Intervening and final magnetic bead cleanups were performed at $1.7 \mathrm{x}$ volume ratio (SPRIselect), and products were eluted in 20 $40 \mu \mathrm{l}$ water.

Barcoding PCR was performed using universal primers to introduce sample-specific barcodes and complete sequencing adaptors, as described previously(14). We used $1 \mathrm{U}$ per reaction of Platinum Taq DNA Polymerase High Fidelity (Invitrogen) in the following buffer: 1.3x Platinum buffer, $0.4 \mathrm{M}$ betaine, $2.5 \mu \mathrm{l} /$ reaction of DMSO, $0.45 \mathrm{mM}$ dNTPs, 1.75 $\mathrm{mM} \mathrm{MgSO}_{4}$, and primers at $0.5 \mu \mathrm{M} .10 \mu \mathrm{l}$ of the product from exponential amplification was used as template, at the following thermocycling conditions: $94{ }^{\circ} \mathrm{C}$ for 2 minutes followed by 15 cycles at $94{ }^{\circ} \mathrm{C}$ for 30 seconds, $56^{\circ} \mathrm{C}$ for 30 seconds, $68{ }^{\circ} \mathrm{C}$ for 1 minute, and a final incubation at $68{ }^{\circ} \mathrm{C}$ for 10 minutes. A final magnetic bead cleanup (SPRIselect) was performed at $1.2 \mathrm{x}$ volume ratio. TARDIS libraries were eluted in $20 \mu \mathrm{l}$ DNA suspension buffer, quantified using fluorometric and electrophoretic assays, and pooled for sequencing. Sequencing was performed on Illumina HiSeq or Illumina NextSeq.

\section{Analysis of TARDIS Sequencing Data}

Paired-end sequencing reads were aligned to human genome hg19 using bwa-mem. Read pairs whose R1 read mapped to the start position of a target primer were considered ontarget reads, and the position of the R2 read was used to determine the length of the template molecule. The UMI sequence and molecule size were used to identify all of the reads that came from the same template molecule. To minimize incorrect assignment of reads to read families, we implemented a directed adjacency graph approach inspired by Smith et al.(44). Briefly, a graph was constructed in which each UMI was a node. An edge from nodeA to 
nodeB was created if their UMIs differ by one base, their DNA molecule size was the same, and nodeA had at least twice as many reads as nodeB. All of the reads from UMIs in each component of the resulting graph constituted a read family and were considered to have come from the same original molecule. UMI variation within a read family was assumed to arise due to PCR or sequencing error. We found that a small number of UMI nodes with very few reads had incoming edges from multiple otherwise separate components. These nodes could not be assigned to a single component unambiguously and reduced the number of independent components in the graph. To resolve this issue, any UMI that had two or more incoming edges and no outgoing edges was removed. We then inferred the allele at the target position by consensus of all R1 reads in a given component, requiring that at least $90 \%$ of the $\mathrm{R} 1$ reads carried a particular allele at the position of interest. In practice, the vast majority of read families contained fewer than 10 reads, and therefore required perfect agreement at the target position. Inferred molecules with less than $90 \%$ read support for any allele were considered inconclusive (mixed RFs).

To ascertain ctDNA detection in a sample, we required support of at least 2 RFs across all mutations covered by at least 100 total RFs. For any mutations supporting ctDNA detection, we required that its $\mathrm{AF}$ (mutant RFs / total RFs) represent at least 0.5 mutant molecules in the reaction. In addition, the ratio between the number of RFs supporting a mutation and mixed RFs observed at that locus had to be $<10$. If only one mutation supported ctDNA detection, we required at least 2 independent RF sizes (to ensure at least two distinct ligated molecules). This requirement was waived if $>1$ mutation supported ctDNA detection. For each mutation observed, the probability of encountering the number and fragment sizes of mutant RFs was calculated using a distribution of background errors (see below). For each sample, the combined probability of mutations detected was calculated and corrected for multiple testing using the Bonferroni approach to account for the number of mutations analyzed in each TARDIS panel. Sample-level ctDNA detection was confirmed if Bonferroni corrected p-value was $<0.05$. To quantify ctDNA in a sample, we calculated mean AFs over all targeted mutations. Because not all sequenced molecules may receive enough reads to form read families, allele fraction (AF) for each mutation was calculated as the proportion of all reads that contained the target variant. For mutations not confidently detected $(<1$ mutant RFs, a ratio of mutant RFs with mixed RFs of $\geq 10$ or $<0.5$ mutant molecules), AFs were set to zero before calculating the sample-level mean ctDNA concentration.

\section{TARDIS Analysis Pipelines}

Target selection and primer design pipelines were developed in Python3 using NumPy, SciPy, networkX, pandas, and matplotlib, and in Julia 0.6.2 using BioJulia, DataFrames, Gadfly, and LightGraphs. Data analysis and plotting were conducted in Python3, Julia 1.1, and R v3 using ggplot2.

\section{Calculation of background error rates}

To measure overall background error rates, we evaluated the first $10 \mathrm{bp}$ from a set of amplicons across multiple representative plasma samples for highest non-reference alleles (starting 3 bp downstream of target-specific primers), excluding the targeted locus. The full 
dataset included 200 loci from each of 39 samples, for a total of 7,800 independent positions. To calculate TARDIS-corrected error rates, we required consensus of all members of an RF, a minimum of 2 RFs with a ratio between variant RFs and mixed RFs $<10$, similar to criteria for detection of individual variants described above. Unlike variant calling for genotyping, TARDIS relies on detection and quantification of pre-identified non-reference alleles at pre-selected genomic loci. We did not call mutations at every sequenced locus and this limited the number of false positives detected. Moreover, given limited template DNA input, individual mutations were not expected at AFs below or close to the background error rate using RF consensus. However, analysis of dozens or hundreds of mutations could result in false positive ctDNA detection due to multiple testing. To ensure confidence in samplelevel ctDNA detection, we built a background distribution for each sample of the number of non-reference RFs and the number of fragment sizes supporting randomly chosen nonreference alleles at non-target loci (3-30 bp from the end of the sequenced primer). We used this distribution to calculate the probability of observing each mutation in a sample. We calculated a combined probability for each sample as the product of probabilities for observed mutations and applied multiple testing correction using the Bonferroni approach, requiring the corrected p-value to be $<0.05$.

\section{Statistical Analysis}

Differences in background error rates, allele fractions and total cfDNA concentration between groups of patients were tested using Wilcoxon rank-sum test. Changes in tumor AFs before and after neoadjuvant therapy were tested using paired Wilcoxon signed-rank test. Correlation between observed and expected AFs in reference samples and between CVs and mutant DNA molecules were evaluated using Pearson correlation coefficient. Two-sided testing was used unless otherwise specified.

\section{Supplementary Material}

Refer to Web version on PubMed Central for supplementary material.

\section{Acknowledgments:}

We thank Marissa Pacheco and Leslie Dixon at Mayo Clinic for their support in collection and processing of patient samples. We thank Josef Herzog at City of Hope for their support in collection and processing of patient samples and clinical data. We thank all the patients for their participation in this study.

Funding: Supported by funding from Ben and Catherine Ivy Foundation to MM, V2015-017 from V Foundation for Cancer Research to MM, BSP-0542-13 from Science Foundation Arizona to MM, charitable donations from SmartPractice and support from by the National Cancer Institute (NCI) of the National Institutes of Health (NIH) under award number 1R01CA223481-01 to MM; The research reported in this publication was also supported by NCI P30CA33572 (Molecular Pathology Core) to TJS, TGen-City of Hope Precision Medicine Pilot Award to MM and TJS, City of Hope Cancer Control and Populations Sciences Pilot award to TJS and JNW, K08CA234394 to TJS, Mayo Development Funds to BAP and Cancer Research UK to CC. The content is solely the responsibility of the authors and does not necessarily represent the official views of the NIH.

\section{References and Notes:}

1. Katz SJ, Jagsi R, Morrow M, Reducing Overtreatment of Cancer With Precision Medicine: Just What the Doctor Ordered. JAMA 319, 1091-1092 (2018). [PubMed: 29470568] 
2. Cortazar P, Zhang L, Untch M, Mehta K, Costantino J, Wolmark N, Bonnefoi H, Cameron D, Gianni L, Valagussa P, Meta-analysis results from the collaborative trials in neoadjuvant breast cancer (CTNeoBC). Cancer Res 72, S1-S11 (2012).

3. Symmans WF, Wei C, Gould R, Yu X, Zhang Y, Liu M, Walls A, Bousamra A, Ramineni M, Sinn B, Hunt K, Buchholz TA, Valero V, Buzdar AU, Yang W, Brewster AM, Moulder S, Pusztai L, Hatzis C, Hortobagyi GN, Long-Term Prognostic Risk After Neoadjuvant Chemotherapy Associated With Residual Cancer Burden and Breast Cancer Subtype. J Clin Oncol 35, 1049-1060 (2017). [PubMed: 28135148]

4. Chagpar AB, Middleton LP, Sahin AA, Dempsey P, Buzdar AU, Mirza AN, Ames FC, Babiera GV, Feig BW, Hunt KK, Kuerer HM, Meric-Bernstam F, Ross MI, Singletary SE, Accuracy of physical examination, ultrasonography, and mammography in predicting residual pathologic tumor size in patients treated with neoadjuvant chemotherapy. Annals of surgery 243, 257-264 (2006). [PubMed: 16432360]

5. Yuan Y, Chen XS, Liu SY, Shen KW, Accuracy of MRI in prediction of pathologic complete remission in breast cancer after preoperative therapy: a meta-analysis. AJR. American journal of roentgenology 195, 260-268 (2010). [PubMed: 20566826]

6. Chaudhuri AA, Chabon JJ, Lovejoy AF, Newman AM, Stehr H, Azad TD, Khodadoust MS, Esfahani MS, Liu CL, Zhou L, Scherer F, Kurtz DM, Say C, Carter JN, Merriott DJ, Dudley JC, Binkley MS, Modlin L, Padda SK, Gensheimer MF, West RB, Shrager JB, Neal JW, Wakelee HA, Loo BW Jr., Alizadeh AA, Diehn M, Early Detection of Molecular Residual Disease in Localized Lung Cancer by Circulating Tumor DNA Profiling. Cancer Discov 7, 1394-1403 (2017). [PubMed: 28899864]

7. Garcia-Murillas I, Schiavon G, Weigelt B, Ng C, Hrebien S, Cutts RJ, Cheang M, Osin P, Nerurkar A, Kozarewa I, Garrido JA, Dowsett M, Reis-Filho JS, Smith IE, Turner NC, Mutation tracking in circulating tumor DNA predicts relapse in early breast cancer. Sci Transl Med 7, 302ra133 (2015).

8. Tie J, Wang Y, Tomasetti C, Li L, Springer S, Kinde I, Silliman N, Tacey M, Wong HL, Christie M, Kosmider S, Skinner I, Wong R, Steel M, Tran B, Desai J, Jones I, Haydon A, Hayes T, Price TJ, Strausberg RL, Diaz LA Jr., Papadopoulos N, Kinzler KW, Vogelstein B, Gibbs P, Circulating tumor DNA analysis detects minimal residual disease and predicts recurrence in patients with stage II colon cancer. Sci Transl Med 8, 346ra392 (2016).

9. Abbosh C, Birkbak NJ, Wilson GA, Jamal-Hanjani M, Constantin T, Salari R, Le Quesne J, Moore DA, Veeriah S, Rosenthal R, Marafioti T, Kirkizlar E, Watkins TBK, McGranahan N, Ward S, Martinson L, Riley J, Fraioli F, Al Bakir M, Gronroos E, Zambrana F, Endozo R, Bi WL, Fennessy FM, Sponer N, Johnson D, Laycock J, Shafi S, Czyzewska-Khan J, Rowan A, Chambers T, Matthews N, Turajlic S, Hiley C, Lee SM, Forster MD, Ahmad T, Falzon M, Borg E, Lawrence D, Hayward M, Kolvekar S, Panagiotopoulos N, Janes SM, Thakrar R, Ahmed A, Blackhall F, Summers Y, Hafez D, Naik A, Ganguly A, Kareht S, Shah R, Joseph L, Marie Quinn A, Crosbie PA, Naidu B, Middleton G, Langman G, Trotter S, Nicolson M, Remmen H, Kerr K, Chetty M, Gomersall L, Fennell DA, Nakas A, Rathinam S, Anand G, Khan S, Russell P, Ezhil V, Ismail B, Irvin-Sellers M, Prakash V, Lester JF, Kornaszewska M, Attanoos R, Adams H, Davies H, Oukrif D, Akarca AU, Hartley JA, Lowe HL, Lock S, Iles N, Bell H, Ngai Y, Elgar G, Szallasi Z, Schwarz RF, Herrero J, Stewart A, Quezada SA, Peggs KS, Van Loo P, Dive C, Lin CJ, Rabinowitz M, Aerts H, Hackshaw A, Shaw JA, Zimmermann BG, consortium TR, consortium P, Swanton C, Phylogenetic ctDNA analysis depicts early-stage lung cancer evolution. Nature 545, 446-451 (2017). [PubMed: 28445469]

10. Rothe F, Silva MJ, Venet D, Campbell C, Bradburry I, Rouas G, de Azambuja E, Maetens M, Fumagalli D, Rodrik-Outmezguine V, Di Cosimo S, Rosa D, Chia S, Wardley A, Ueno T, Janni W, Huober J, Baselga J, Piccart M, Loi S, Sotiriou C, Dawson SJ, Ignatiadis M, Circulating Tumor DNA in HER2-Amplified Breast Cancer: A Translational Research Substudy of the NeoALTTO Phase III Trial. Clinical cancer research : an official journal of the American Association for Cancer Research 25, 3581-3588 (2019). [PubMed: 30862692]

11. Tie J, Cohen JD, Wang Y, Li L, Christie M, Simons K, Elsaleh H, Kosmider S, Wong R, Yip D, Lee M, Tran B, Rangiah D, Burge M, Goldstein D, Singh M, Skinner I, Faragher I, Croxford M, Bampton C, Haydon A, Jones IT, C SK, Price T, Schaefer MJ, Ptak J, Dobbyn L, Silliman N, Kinde I, Tomasetti C, Papadopoulos N, Kinzler K, Volgestein B, Gibbs P, Serial circulating tumour 
DNA analysis during multimodality treatment of locally advanced rectal cancer: a prospective biomarker study. Gut 68, 663-671 (2019). [PubMed: 29420226]

12. Riva F, Bidard FC, Houy A, Saliou A, Madic J, Rampanou A, Hego C, Milder M, Cottu P, Sablin MP, Vincent-Salomon A, Lantz O, Stern MH, Proudhon C, Pierga JY, Patient-Specific Circulating Tumor DNA Detection during Neoadjuvant Chemotherapy in Triple-Negative Breast Cancer. Clin Chem 63, 691-699 (2017). [PubMed: 28073896]

13. Stover DG, Parsons HA, Ha G, Freeman SS, Barry WT, Guo H, Choudhury AD, Gydush G, Reed SC, Rhoades J, Rotem D, Hughes ME, Dillon DA, Partridge AH, Wagle N, Krop IE, Getz G, Golub TR, Love JC, Winer EP, Tolaney SM, Lin NU, Adalsteinsson VA, Association of Cell-Free DNA Tumor Fraction and Somatic Copy Number Alterations With Survival in Metastatic TripleNegative Breast Cancer. J Clin Oncol 36, 543-553 (2018). [PubMed: 29298117]

14. Murtaza M, Dawson SJ, Pogrebniak K, Rueda OM, Provenzano E, Grant J, Chin SF, Tsui DW, Marass F, Gale D, Ali HR, Shah P, Contente-Cuomo T, Farahani H, Shumansky K, Kingsbury Z, Humphray S, Bentley D, Shah SP, Wallis M, Rosenfeld N, Caldas C, Multifocal clonal evolution characterized using circulating tumour DNA in a case of metastatic breast cancer. Nat Commun 6, 8760 (2015). [PubMed: 26530965]

15. Forshew T, Murtaza M, Parkinson C, Gale D, Tsui DW, Kaper F, Dawson SJ, Piskorz AM, Jimenez-Linan M, Bentley D, Hadfield J, May AP, Caldas C, Brenton JD, Rosenfeld N, Noninvasive identification and monitoring of cancer mutations by targeted deep sequencing of plasma DNA. Sci Transl Med 4, 136ra168 (2012).

16. Kinde I, Wu J, Papadopoulos N, Kinzler KW, Vogelstein B, Detection and quantification of rare mutations with massively parallel sequencing. Proceedings of the National Academy of Sciences of the United States of America 108, 9530-9535 (2011). [PubMed: 21586637]

17. Blum Murphy M, Xiao L, Patel VR, Maru DM, Correa AM, F GA, Liao Z, Komaki R, Lin SH, Skinner HD, Vaporciyan A, Walsh GL, Swisher SG, Sepesi B, Lee JH, Bhutani MS, Weston B, Hofstetter WL, Ajani JA, Pathological complete response in patients with esophageal cancer after the trimodality approach: The association with baseline variables and survival-The University of Texas MD Anderson Cancer Center experience. Cancer 123, 4106-4113 (2017). [PubMed: 28885712]

18. Cercek A, Goodman KA, Hajj C, Weisberger E, Segal NH, Reidy-Lagunes DL, Stadler ZK, Wu AJ, Weiser MR, Paty PB, Guillem JG, Nash GM, Temple LK, Garcia-Aguilar J, Saltz LB, Neoadjuvant chemotherapy first, followed by chemoradiation and then surgery, in the management of locally advanced rectal cancer. J Natl Compr Canc Netw 12, 513-519 (2014). [PubMed: 24717570]

19. Bettegowda C, Sausen M, Leary RJ, Kinde I, Wang Y, Agrawal N, Bartlett BR, Wang H, Luber B, Alani RM, Antonarakis ES, Azad NS, Bardelli A, Brem H, Cameron JL, Lee CC, Fecher LA, Gallia GL, Gibbs P, Le D, Giuntoli RL, Goggins M, Hogarty MD, Holdhoff M, Hong SM, Jiao Y, Juhl HH, Kim JJ, Siravegna G, Laheru DA, Lauricella C, Lim M, Lipson EJ, Marie SK, Netto GJ, Oliner KS, Olivi A, Olsson L, Riggins GJ, Sartore-Bianchi A, Schmidt K, Shih 1 M, Oba-Shinjo SM, Siena S, Theodorescu D, Tie J, Harkins TT, Veronese S, Wang TL, Weingart JD, Wolfgang CL, Wood LD, Xing D, Hruban RH, Wu J, Allen PJ, Schmidt CM, Choti MA, Velculescu VE, Kinzler KW, Vogelstein B, Papadopoulos N, Diaz LA Jr., Detection of circulating tumor DNA in early- and late-stage human malignancies. Sci Transl Med 6, 224 ra224 (2014).

20. Dawson SJ, Tsui DW, Murtaza M, Biggs H, Rueda OM, Chin SF, Dunning MJ, Gale D, Forshew T, Mahler-Araujo B, Rajan S, Humphray S, Becq J, Halsall D, Wallis M, Bentley D, Caldas C, Rosenfeld N, Analysis of circulating tumor DNA to monitor metastatic breast cancer. N Engl J Med 368, 1199-1209 (2013). [PubMed: 23484797]

21. Butler TM, Boniface CT, Johnson-Camacho K, Tabatabaei S, Melendez D, Kelley T, Gray J, Corless CL, Spellman PT, Circulating tumor DNA dynamics using patient-customized assays are associated with outcome in neoadjuvantly treated breast cancer. Cold Spring Harb Mol Case Stud 5, (2019).

22. Magbanua M, Brown-Swigart L, Hirst G, Yau C, Wolf D, Ma A, Bergin E, Venters S, Sethi H, Wu H-T, Salari R, Tin T, Sawyer S, Louie M, Zimmermann B, Lin C-H, Keats J, Liang W, Cuyugan L, Enriquez D, Tripathy D, Chien A, Forero A, DeMichele A, Liu M, Delson A, Asare S, Esserman L, van't Veer L, Abstract PD2-01: Personalized serial circulating tumor DNA (ctDNA) analysis in 
high-risk early stage breast cancer patients to monitor and predict response to neoadjuvant therapy and outcome in the I-SPY 2 TRIAL. Cancer Research 79, PD2-01-PD02-01 (2019).

23. Anagnostou V, Forde PM, White JR, Niknafs N, Hruban C, Naidoo J, Marrone K, Sivakumar IKA, Bruhm DC, Rosner S, Phallen J, Leal A, Adleff V, Smith KN, Cottrell TR, Rhymee L, Palsgrove DN, Hann CL, Levy B, Feliciano J, Georgiades C, Verde F, Illei P, Li QK, Gabrielson E, Brock MV, Isbell JM, Sauter JL, Taube J, Scharpf RB, Karchin R, Pardoll DM, Chaft JE, Hellmann MD, Brahmer JR, Velculescu VE, Dynamics of Tumor and Immune Responses during Immune Checkpoint Blockade in Non-Small Cell Lung Cancer. Cancer Res 79, 1214-1225 (2019). [PubMed: 30541742]

24. Phallen J, Sausen M, Adleff V, Leal A, Hruban C, White J, Anagnostou V, Fiksel J, Cristiano S, Papp E, Speir S, Reinert T, Orntoft MW, Woodward BD, Murphy D, Parpart-Li S, Riley D, Nesselbush M, Sengamalay N, Georgiadis A, Li QK, Madsen MR, Mortensen FV, Huiskens J, Punt C, van Grieken N, Fijneman R, Meijer G, Husain H, Scharpf RB, Diaz LA Jr., Jones S, Angiuoli S, Orntoft T, Nielsen HJ, Andersen CL, Velculescu VE, Direct detection of early-stage cancers using circulating tumor DNA. Sci Transl Med 9, (2017).

25. Stahlberg A, Krzyzanowski PM, Jackson JB, Egyud M, Stein L, Godfrey TE, Simple, multiplexed, PCR-based barcoding of DNA enables sensitive mutation detection in liquid biopsies using sequencing. Nucleic Acids Res 44, e105 (2016). [PubMed: 27060140]

26. Narayan A, Carriero NJ, Gettinger SN, Kluytenaar J, Kozak KR, Yock TI, Muscato NE, Ugarelli P, Decker RH, Patel AA, Ultrasensitive measurement of hotspot mutations in tumor DNA in blood using error-suppressed multiplexed deep sequencing. Cancer Res 72, 3492-3498 (2012). [PubMed: 22581825]

27. Zviran A, Hill ST, Schulman R, Shah M, Deochand S, Ha G, Reed S, Rotem D, Gydush G, Rhoades J, Huang K, Liao W, Maloney D, Omans N, Malbari M, Spinelli CF, Kazancioglu S, Robine N, Adalsteinsson V, Houck-Loomis B, Altorki N, Landau DA, Abstract 3247: Genomewide cell-free DNA mutation integration for sensitive cancer detection. Cancer Research 78, 3247-3247 (2018).

28. Ruiz-Valdepenas A, Heider K, Doughton G, Qian W, Massie C, Chandrananda D, Smith C, Gale D, Moseley E, Castedo C, Stone A, Thorbinson C, Eisen T, Rassl D, Harden S, Rintoul R, Rosenfeld N, MA 11.02 Circulating Tumor DNA in Early Stage NSCLC: High Sensitivity Analysis in Low Burden Disease. LUCID Study Update. Journal of Thoracic Oncology 12, S1843-S1844 (2017).

29. Cohen JD, Li L, Wang Y, Thoburn C, Afsari B, Danilova L, Douville C, Javed AA, Wong F, Mattox A, Hruban RH, Wolfgang CL, Goggins MG, Dal Molin M, Wang TL, Roden R, Klein AP, Ptak J, Dobbyn L, Schaefer J, Silliman N, Popoli M, Vogelstein JT, Browne JD, Schoen RE, Brand RE, Tie J, Gibbs P, Wong HL, Mansfield AS, Jen J, Hanash SM, Falconi M, Allen PJ, Zhou S, Bettegowda C, Diaz LA Jr., Tomasetti C, Kinzler KW, Vogelstein B, Lennon AM, Papadopoulos $\mathrm{N}$, Detection and localization of surgically resectable cancers with a multi-analyte blood test. Science 359, 926-930 (2018). [PubMed: 29348365]

30. Sparano JA, Gray RJ, Makower DF, Pritchard KI, Albain KS, Hayes DF, Geyer CE Jr., Dees EC, Goetz MP, Olson JA Jr., Lively T, Badve SS, Saphner TJ, Wagner LI, Whelan TJ, Ellis MJ, Paik S, Wood WC, Ravdin PM, Keane MM, Gomez Moreno HL, Reddy PS, Goggins TF, Mayer IA, Brufsky AM, Toppmeyer DL, Kaklamani VG, Berenberg JL, Abrams J, Sledge GW Jr., Adjuvant Chemotherapy Guided by a 21-Gene Expression Assay in Breast Cancer. N Engl J Med 379, $111-$ 121 (2018). [PubMed: 29860917]

31. Markus H, Contente-Cuomo T, Farooq M, Liang WS, Borad MJ, Sivakumar S, Gollins S, Tran NL, Dhruv HD, Berens ME, Bryce A, Sekulic A, Ribas A, Trent JM, LoRusso PM, Murtaza M, Evaluation of pre-analytical factors affecting plasma DNA analysis. Sci Rep 8, 7375 (2018). [PubMed: 29743667]

32. Li H, Aligning sequence reads, clone sequences and assembly contigs with BWA-MEM. arXiv preprint arXiv:1303.3997, (2013).

33. Van der Auwera GA, Carneiro MO, Hartl C, Poplin R, Del Angel G, Levy-Moonshine A, Jordan T, Shakir K, Roazen D, Thibault J, Banks E, Garimella KV, Altshuler D, Gabriel S, DePristo MA, From FastQ data to high confidence variant calls: the Genome Analysis Toolkit best practices pipeline. Curr Protoc Bioinformatics 43, 1110 11-33 (2013). [PubMed: 25431634] 
34. Garrison E, Marth G, Haplotype-based variant detection from short-read sequencing. ArXiv eprints. 2012.

35. Cibulskis K, Lawrence MS, Carter SL, Sivachenko A, Jaffe D, Sougnez C, Gabriel S, Meyerson M, Lander ES, Getz G, Sensitive detection of somatic point mutations in impure and heterogeneous cancer samples. Nat Biotechnol 31, 213-219 (2013). [PubMed: 23396013]

36. Christoforides A, Carpten JD, Weiss GJ, Demeure MJ, Von Hoff DD, Craig DW, Identification of somatic mutations in cancer through Bayesian-based analysis of sequenced genome pairs. BMC Genomics 14, 302 (2013). [PubMed: 23642077]

37. Saunders CT, Wong WS, Swamy S, Becq J, Murray LJ, Cheetham RK, Strelka: accurate somatic small-variant calling from sequenced tumor-normal sample pairs. Bioinformatics 28, 1811-1817 (2012). [PubMed: 22581179]

38. Favero F, Joshi T, Marquard AM, Birkbak NJ, Krzystanek M, Li Q, Szallasi Z, Eklund AC, Sequenza: allele-specific copy number and mutation profiles from tumor sequencing data. Ann Oncol 26, 64-70 (2015). [PubMed: 25319062]

39. McGranahan N, Favero F, de Bruin EC, Birkbak NJ, Szallasi Z, Swanton C, Clonal status of actionable driver events and the timing of mutational processes in cancer evolution. Sci Transl Med 7, 283 ra254 (2015).

40. Roth A, Khattra J, Yap D, Wan A, Laks E, Biele J, Ha G, Aparicio S, Bouchard-Cote A, Shah SP, PyClone: statistical inference of clonal population structure in cancer. Nat Methods 11, 396-398 (2014). [PubMed: 24633410]

41. Untergasser A, Cutcutache I, Koressaar T, Ye J, Faircloth BC, Remm M, Rozen SG, Primer3--new capabilities and interfaces. Nucleic Acids Res 40, e115 (2012). [PubMed: 22730293]

42. Kielbasa SM, Wan R, Sato K, Horton P, Frith MC, Adaptive seeds tame genomic sequence comparison. Genome Res 21, 487-493 (2011). [PubMed: 21209072]

43. Kwok CK, Ding Y, Sherlock ME, Assmann SM, Bevilacqua PC, A hybridization-based approach for quantitative and low-bias single-stranded DNA ligation. Anal Biochem 435, 181-186 (2013). [PubMed: 23399535]

44. Smith T, Heger A, Sudbery I, UMI-tools: modeling sequencing errors in Unique Molecular Identifiers to improve quantification accuracy. Genome Res 27, 491-499 (2017). [PubMed: 28100584] 
A)

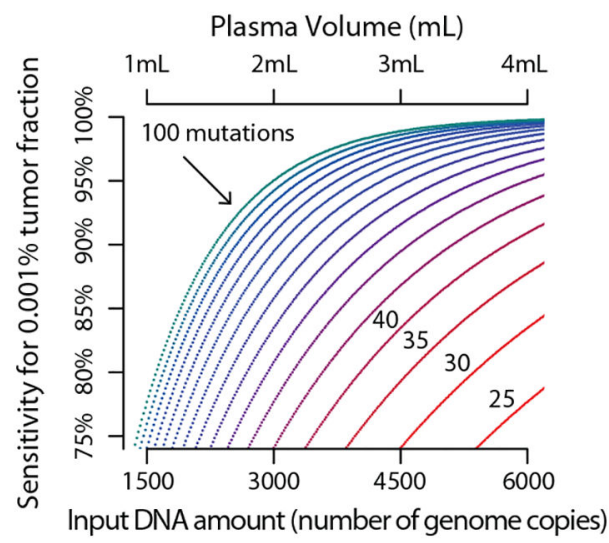

B)
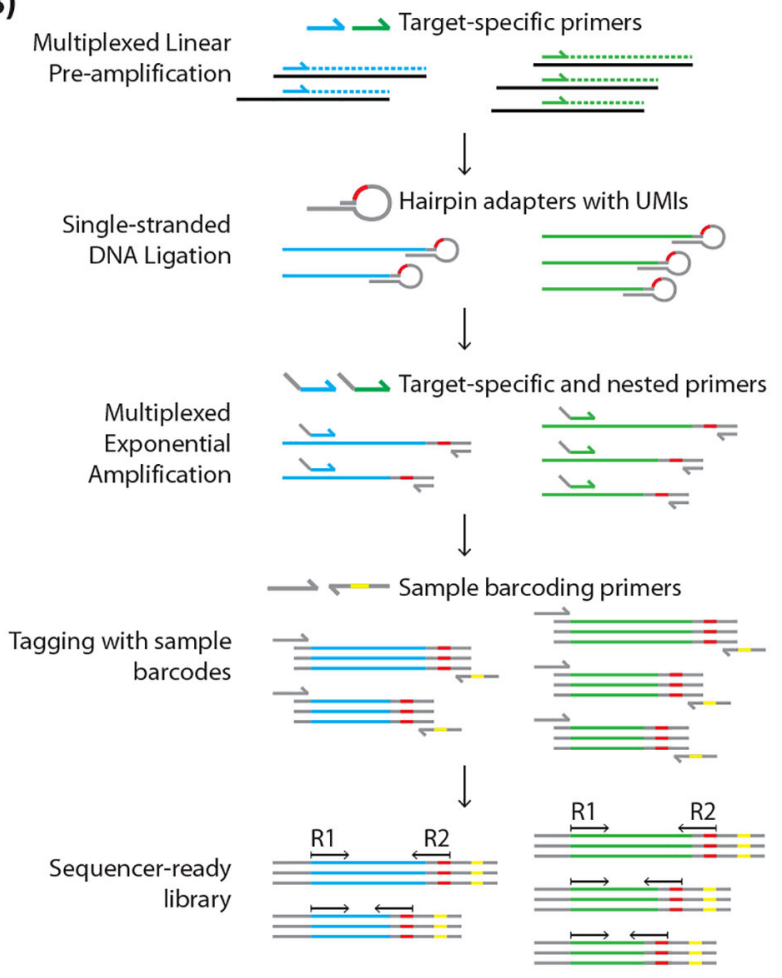

$\longrightarrow \sim-$ Sample barcoding primers

Multiplexed Exponential
C)

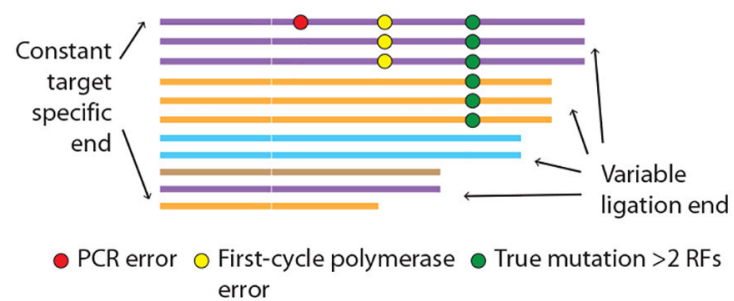

Fig. 1.

Development of a multiplexed assay for personalized ctDNA detection and monitoring. (A)

Results of binomial sampling at varying input DNA amounts (bottom $\mathrm{x}$-axis) and corresponding plasma volumes (top x-axis). Maximum theoretical sensitivity for 1 in $10^{5}$ tumor fraction (y-axis) was calculated as the probability of detecting at least 1 mutated DNA fragment for at least 1 targeted mutation. Each line shows the number of mutations tested (25 to 100, increments of 5). A plasma DNA concentration of $5 \mathrm{ng} / \mathrm{ml}$ plasma (or 1500 haploid genome copies) and no molecular loss during library preparation is assumed. Sensitivity for detection of ctDNA at $0.001 \%$ tumor fraction is limited if only $2-4$ mutations are assayed but can be improved with higher input of plasma DNA and increasing number of patient-specific mutations. (B) For TARDIS, sequencing library preparation includes linear pre-amplification to improve molecular conversion, single-stranded DNA ligation using hairpin oligonucleotides to allow error suppression using template fragment sizes and unique molecular identifiers (UMIs), and multiplexed PCR to enrich targeted genomic loci. (C) Schematic representation of read structure and error suppression using TARDIS. TARDIS uses UMIs (indicated by different read colors) and fragment sizes to group sequencing reads into read families. We exclude PCR errors (red circle) by requiring consensus of all RF members and polymerase errors (yellow circles) introduced during linear pre-amplification by requiring support by at least 2 RFs. Additional description of error suppression strategies is provided in the Materials and Methods. 
A) Expected Allele Fraction in Reference Samples $(n=93)$

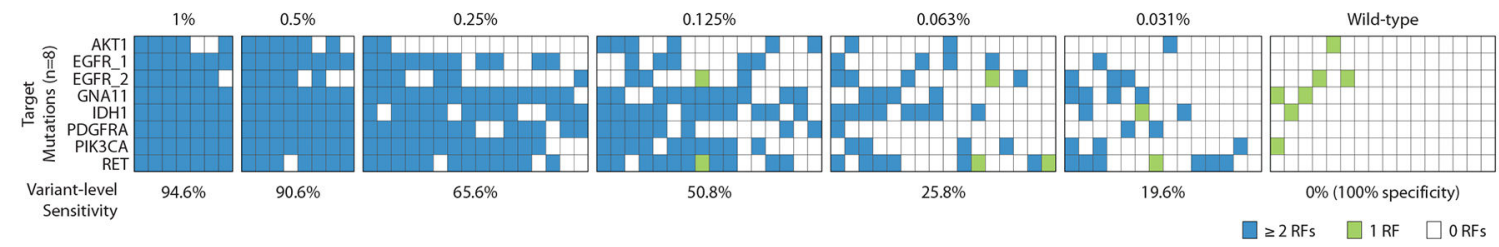

B)

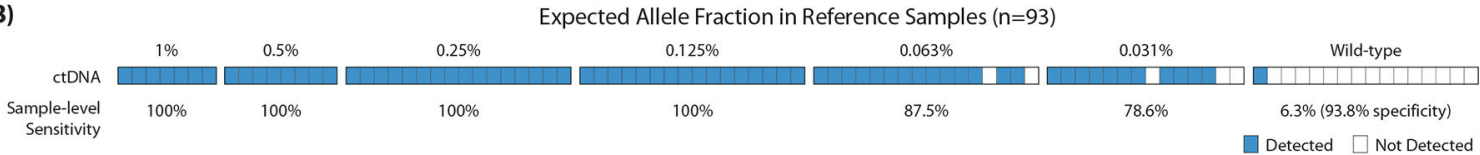

C)

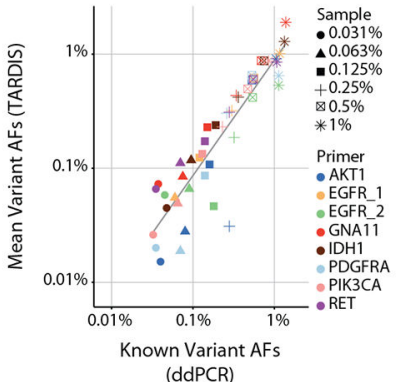

D)

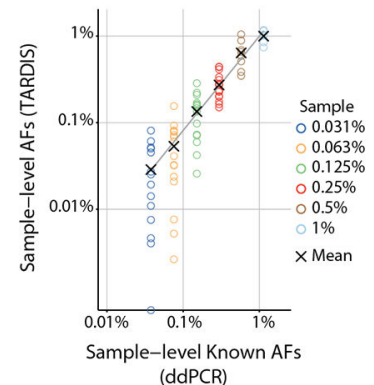

E)

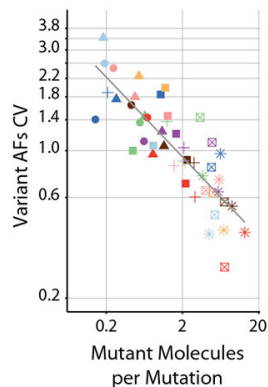

F)

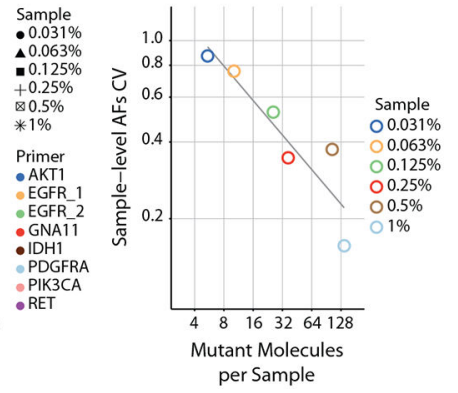

Fig. 2.

Analytical performance of TARDIS in reference samples. (A) Mutation-level sensitivity and specificity across 93 reference samples and 8 mutations, requiring each mutation to be supported by $\geq 2$ RFs and an AF consistent with $\searrow 0.5$ mutant molecules. Each row corresponds to a targeted mutation and each column corresponds to a single sample analyzed at the identified AF. (B) Sample-level sensitivity and specificity, requiring $\geq 2$ RFs contributed by one mutation with multiple fragment sizes or $>1$ mutations, each with an AF consistent with $\searrow 0.5$ mutant molecules. (C) Comparison of variant AFs observed using TARDIS (y-axis) with expected variant AFs measured using ddPCR (x-axis, 48 data points). For each variant, mean observed AF across all replicates (at the same expected AF) is presented,. Gray line is linear fit. (D) Comparison of sample AFs observed using TARDIS (mean for all 8 mutations assayed in each replicate sample, 77 data points) with known sample AFs (mean of known variant AFs). Gray line is linear fit to the mean at each expected AF. (E) CVs of variant AFs decreased with increasing number of mutant molecules per mutation. CVs calculated across 7-16 replicates at each mutation fraction for each of 8 mutations (48 data points). (F) CVs of sample-level AFs were lower than those for individual mutations, demonstrating the advantage of leveraging multiple mutations for ctDNA quantification. CVs calculated across 7-16 replicates for sample-level AFs across 6 mutation fractions. 
A) Expected Allele Fraction in Reference Samples $(n=56)$
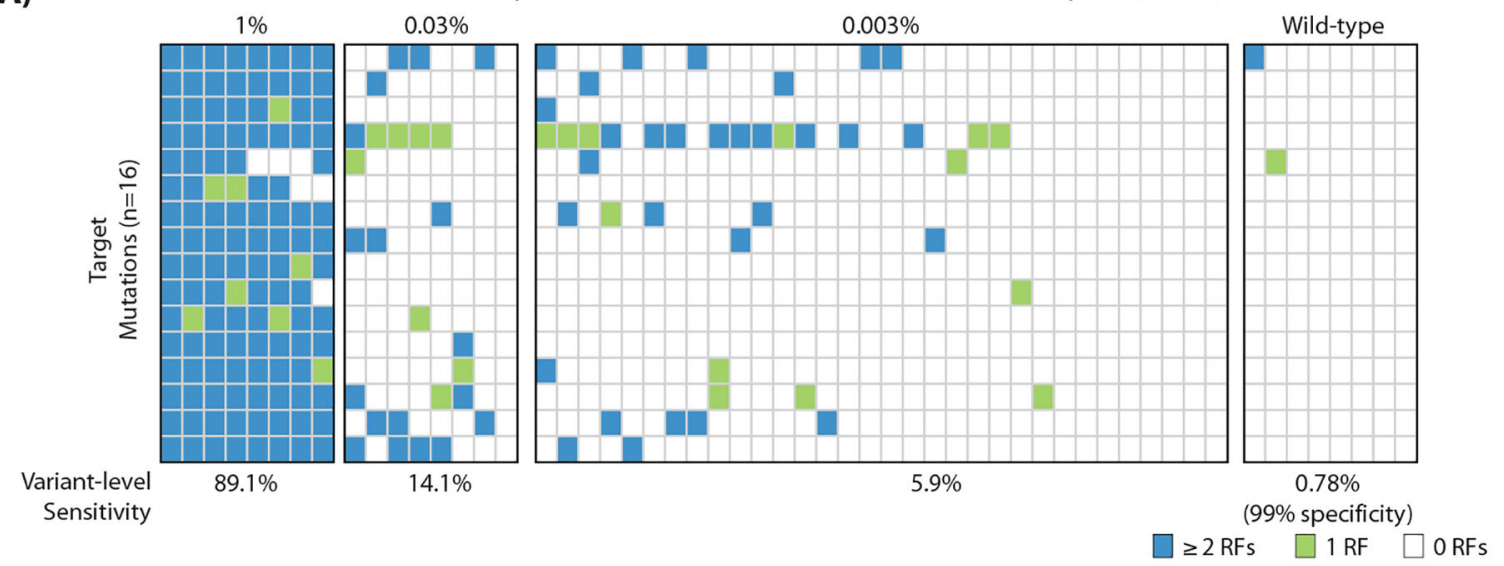

B)
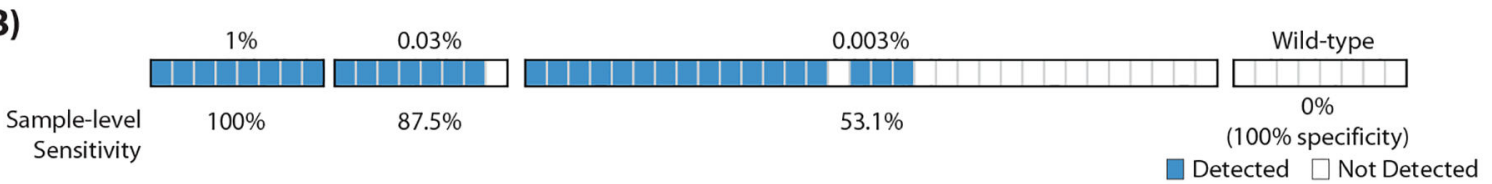

C)

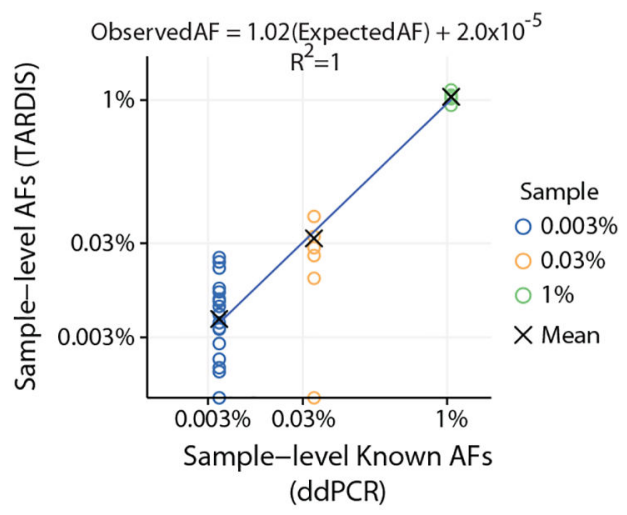

D)

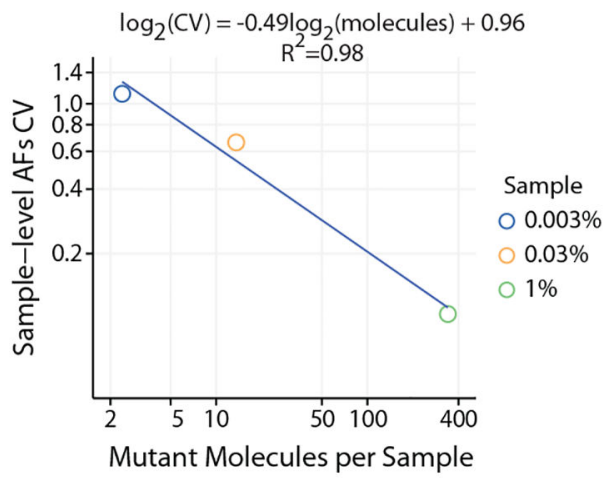

Fig. 3.

Evaluation of analytical performance in reference samples at 3 in $10^{5}$ tumor fraction. (A) Variant-level sensitivity and specificity across 56 reference samples and 16 mutations, requiring each mutation to be supported by $\geq 2 \mathrm{RFs}$ and an AF consistent with $\searrow 0.5$ mutant molecules. 22 mutations were analyzed in this experiment. However, 6 mutations were inferred to contribute biological background because these were recurrently observed in a wild-type DNA sample sourced from immortalized cell lines. These included known hotspot variants in TP53 ( $n=4 / 4$ targets), APC ( $n=1 / 2$ targets), and GNAS ( $n=1 / 1$ targets). These mutations were dropped from further analysis. Each row corresponds to a targeted mutation and each column corresponds to a single sample analyzed at the identified AF. (B) Samplelevel sensitivity and specificity, requiring $\geq 2$ RFs contributed by one mutation with multiple sizes or $>1$ mutations, each with an AF consistent with $\searrow 0.5$ mutant molecules. Although a mutation with 2 RFs was observed in 1 wild-type sample, this mutation was supported by a single size and at the sample-level, ctDNA was determined to be undetectable. (C) Accuracy evaluated by comparison of sample AFs observed using TARDIS (mean for all 16 mutations assayed in each replicate sample) with known sample AFs (mean of known variant AFs 
measured using digital PCR). Blue line is linear fit to the mean at each expected AF. (D) Precision evaluated using CVs of sample-level AFs, calculated across 8-32 replicates. 
A)
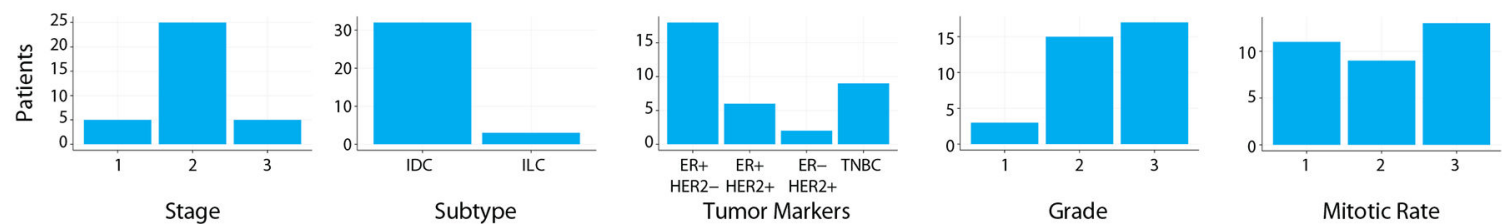

B)

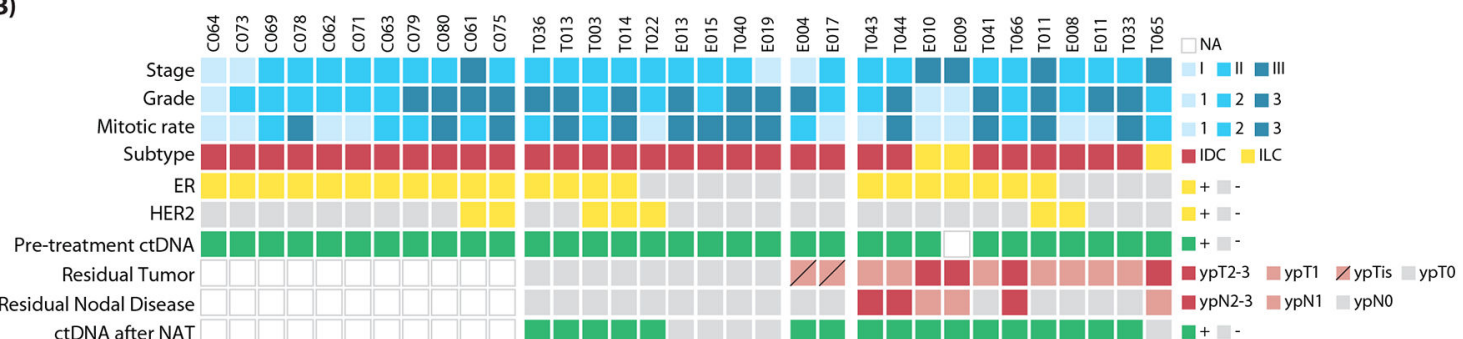
ctDNA after NAT

C)

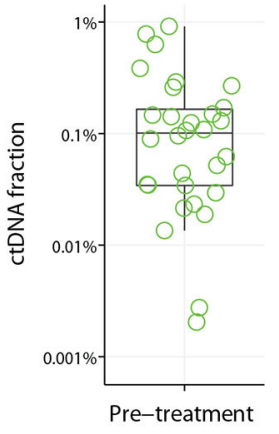

D)

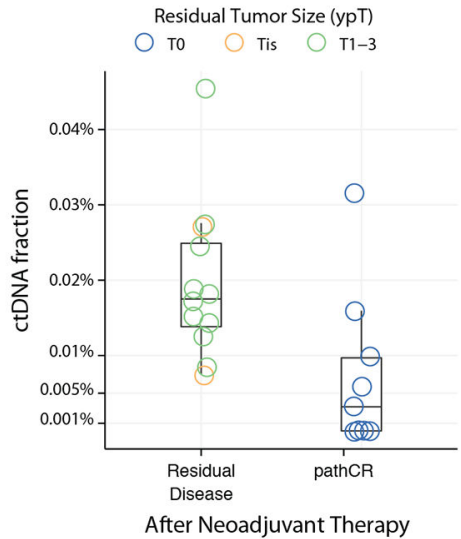

E)

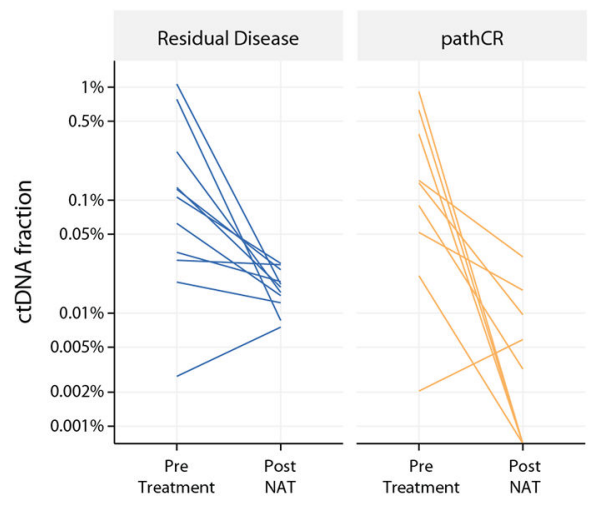

Fig. 4.

ctDNA analysis in patients with early and locally advanced breast cancer before treatment and after completion of neoadjuvant therapy. (A) Clinical characteristics of the cohort. (B) Summary of results, tumor stage, grade, mitotic rate, subtype, ctDNA detection before treatment and after neoadjuvant therapy and residual disease assessment. Pathological TNM staging was performed after surgery and completion of NAT. NA: not available or applicable, IDC: invasive ductal carcinoma, ILC: invasive lobular carcinoma, ypTis: in situ disease, ypT1-3 and ypN1-3, tumor and nodal stage upon pathological staging. (C) ctDNA fraction at baseline. (D). ctDNA fraction after completion of neoadjuvant therapy, grouped by clinical response to treatment (residual disease vs. pathological complete response). (E) Changes in pre- and post-treatment ctDNA fraction in patients with residual disease and pathCR. 


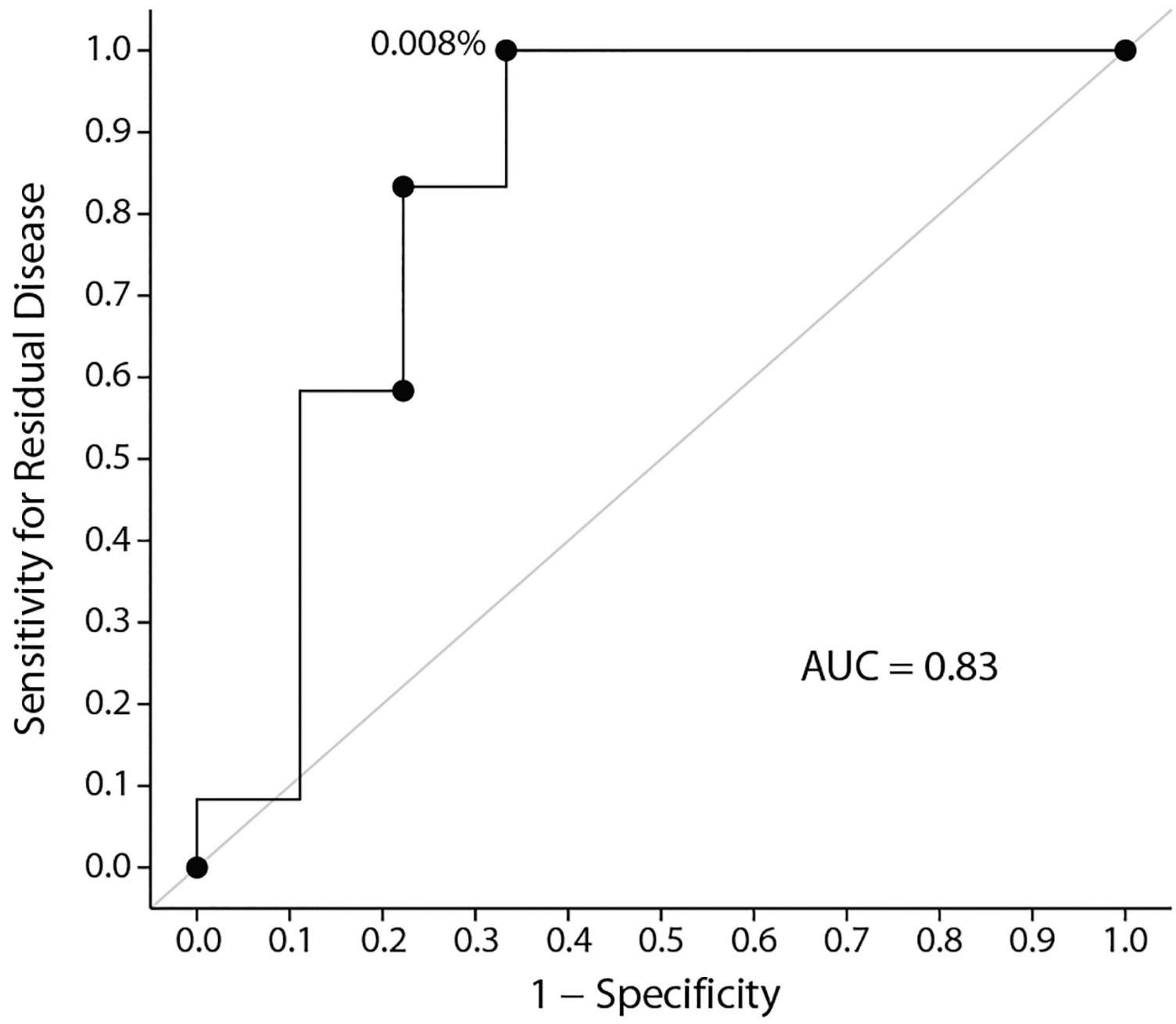

Fig. 5.

Receiver operating characteristic curve for predicting residual disease using ctDNA fraction after completion of neoadjuvant therapy. 